\title{
Recognition of On-line Hand Sketched Complex Diagrams
}

\author{
A. Al-Marakeby \\ Systems and Computers Engineering Dept, Faculty of Engineering, Al-Azhar University, Cairo, Egypt
}

\begin{abstract}
The spread and availability of pen-based devices increased the research in free handwriting and hand sketched recognition. Most of systems in hand sketched diagram recognition are limited to a specific application or limited to specific shapes. Complex diagrams recognition requires not only understanding the primitive shapes but also determining relations between these shapes. This work presents a system for recognition of hand-sketched complex diagrams which create active and intelligent relations between different diagram components. A multi-layers framework is developed which consists of stroke layer, shape layer, and diagram layer. A distance function is used to measure the similarity between template shapes and user sketched shapes. In the diagram layer, many adjustments are applied to enhance the positions and sizes of shapes, increasing the harmony and regularity of shapes arrangement. This system has been tested using a set of primitive shapes and a set of complex diagram and high accuracy results have been obtained.
\end{abstract}

Keywords: Diagram recognition, free hand sketch, feature extraction, pattern recognition.

\section{INTRODUCTION}

Most of documents consist mainly of text and diagrams. Using standard software tools to create diagrams familiar pencil-and-paper process, and transform the freerepresents a tedious work for users. With the widespread hand sketches into the symbols of the domain-specific of touch screens in mobile phones, tablets and PCs, users knowledge. T. Hammond et.al. designed A Sketch prefer to create documents by freehand using stylus or Recognition System for Recognizing Free-Hand Course of fingers. Sketching diagrams by free hand is very fast, easy, Action Diagrams[10]. This system is tested on 5,900 handand user friendly, but usually they have bad appearance. drawn symbols, and accuracy of $90 \%$ is achieved when Also hand-sketched diagrams and drawings are not considering the top 3 interpretations. understandable for software tools such as CAD systems. Sketch recognition systems are developed to interpret and understand hand sketched diagrams and drawings, converting them into standard, nice, and understandable shapes. The recognition of hand drawings has a variety of uses, including symbol recognition in Computer-Aided Design systems, providing automatic correction or understanding of diagrams for immediate feedback in educational settings, functioning as alternative inputs for small keyboard-less devices (such as Palm Pilots), or providing gestural interfaces[2][9][10]. A lot of research has been done in recognition of handwritten characters while hand sketched diagram recognition still needs more improvements and progress. There have been a wide variety of sketch recognition techniques[3][4][5]. Most of them are primitives-based, where the inputting patterns are first decomposed into basic geometric primitives (such as lines and curves) and then assembled into a graphical structure that encodes both the intrinsic attributes of the primitives and their relationships [12]. Andreas et. al developed a method for the recognition of on-line handwritten commutative diagrams. Diagrams are formed with arrows that join relatively simple mathematical expressions [1].Zhenming and Guichao presented a Sketch Recognition based Intelligent Whiteboard Teaching System[14]. Their system can recognize the

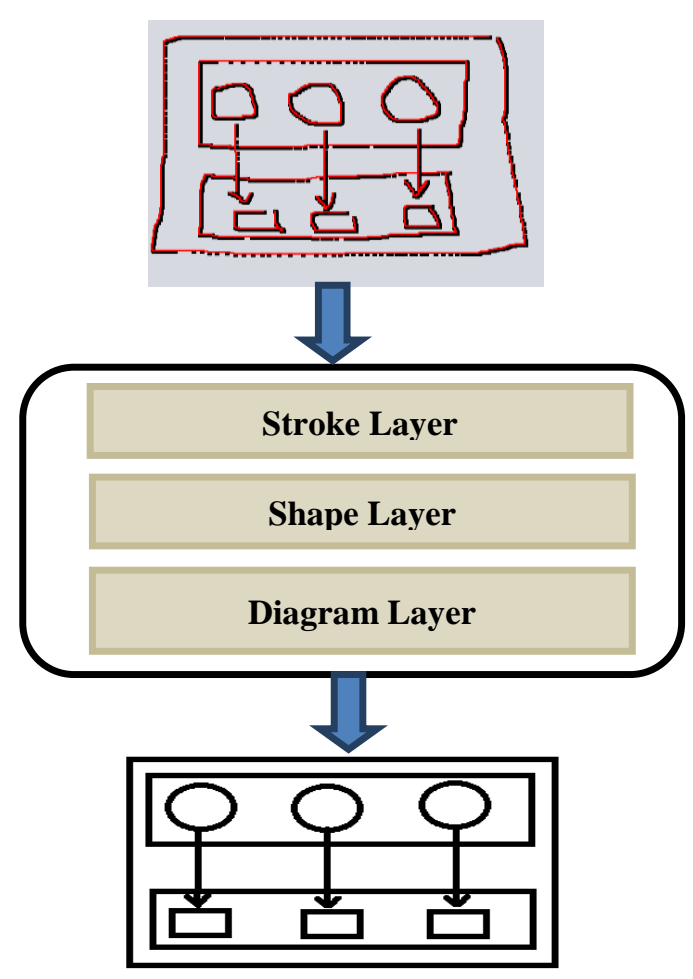

Fig.1 Recognition System 
Most of sketch recognition systems are limited to a to classify the shapes to one of a set of specified classes specific application or limited to specific shapes. Also the [11][13][14]. There is another important problem despite grouping of primitive shapes in a single diagram has many the used technique or method. This problem is related to problems and challenges. Most of research consider the the number of shape strokes, where most of researchers problem of recognition of primitive shapes in depth, but simplify the problem by the assumption of single stroke pay less attention for the grouping problem. For example, shapes. This problem is discussed later in this section. a diagram consists of 3 adjacent circles will be interpreted correctly by most of sketch recognition systems. If the user want to draw the circles with same radius and same distances between centres, it is very difficult for him to adjust that by hand. Recognition systems don't take into account such issues and the result interpreted diagram looks like distorted one in many cases. .In this system, a multi-layers framework is developed which take into consideration these problems. It consists of three layers: stroke layer, shape layer, and diagram layers as shown in fig.1.

\section{STROKES PRE-PROCESSING}

In off-line recognition the sketch is available as an image, while in the on-line case the two dimensional coordinates of successive points are available in order as a function of time [7]. Usually on-line recognition accuracy is better than off-line accuracy due to the availability ofmore information about strokes order and speed. Stroke requires some pre-processing steps to facilitate the recognition of shapes.

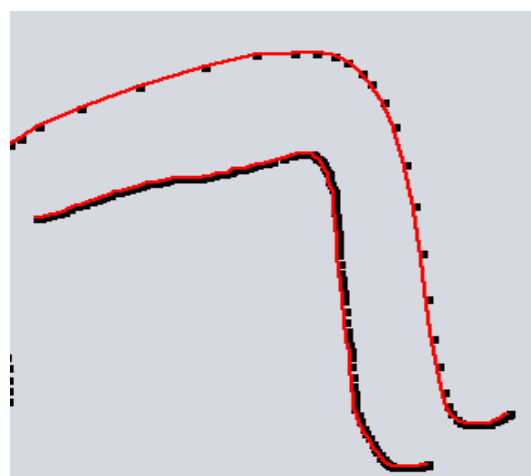

Fig. 2 Strokepre-processing

A simple filling method is used to prepare the strokes for further processing. As shown in fig.2 the stroke has fewer points when it is drawn with fast speed. The red stroke represents the stroke after connecting points. After this step, features points are extracted, and the stroke is segmented into an initial approximations lines. These features are based on maximum curvature points. Fig. 3 shows this initial approximation.

\section{III.SHAPE RECOGNITION}

Many different shape recognition techniques are developed. Some methods depends on linear least squares fitting to a conic section equation, so it can identify sketch strokes, lines, elliptic arcs, and corners [6]. Other methods depend on Fuzzy logic, neural networks or SVM are used

\section{A. Distance function}

In this work a distance function is derived to measure the similarity between the template shape and the sketched strokes. The result recognized shape is the minimum value of distances of all templates. Algorithm 1. Illustrate a simplification for the recognition process.

\section{Algorithm.1: \\ For all templates \\ Estimate initial template parameters (location and size) \\ Measure the distance between template and sketched shape \\ Initial Hypothesis Rejection \\ Iterate with small parameter variations until convergence \\ Measure the minimum distance for this template \\ next template \\ The result shape is the minimum distance for all templates}

The distance between a template and sketched shape is given in equation (1).

\section{Dist $\left(\right.$ tempalte $_{\mathbf{j}}$, Sketch $\left._{\mathbf{i}}\right)=$ \\ a * Dist_ShapeToTemp+b* Dist_TempToShape}

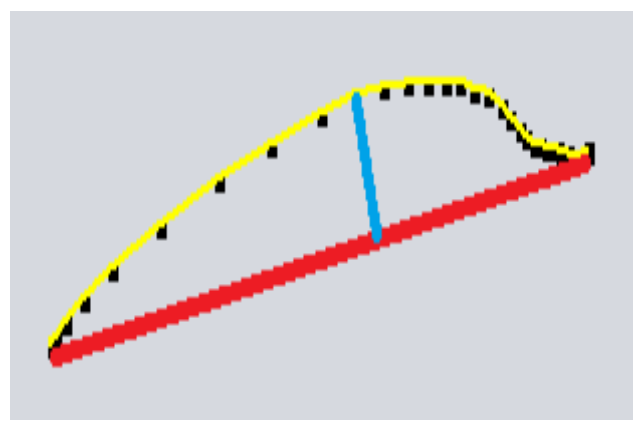

Fig. 3Line approximation

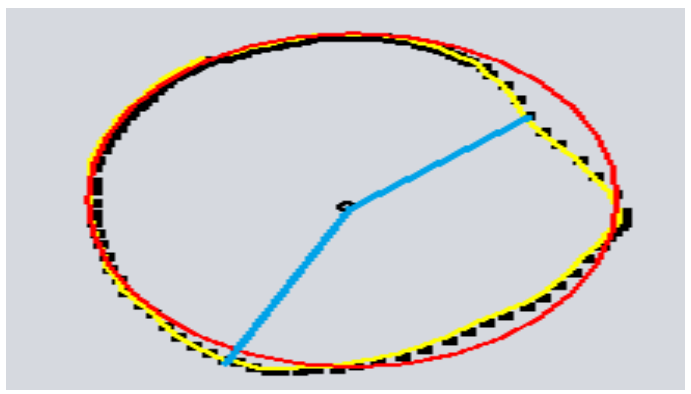

Fig. 4 Distance function 
Vol. 5, Issue 12, December 2016

The shape to template distance is the sum of all stroke point distances as given in equation (2). While the number of stroke points depends on the drawing speed, the normalized stroke points by filling in between points is used as mentioned in section 2 .

$$
\text { ShapeToTemplate }_{\text {dist }}=\sum_{\mathrm{i}=1}^{\mathrm{n}} \operatorname{Dist}\left(\mathbf{P}_{\mathrm{i}}\right)
$$

The term "Dist_TemplateToShape" in equation (2) represents the covering of strokes to all template components. For example, an arc has a zero distance to a specific circle but don't cover the complete circle circumference. This problem is solved by adding the second term in equation (2) with constants $a, b$.

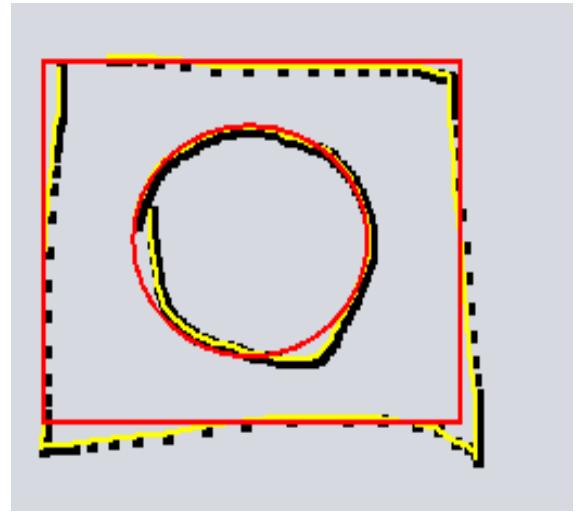

Fig. 5 shape recognition

The "initial template parameters" step is important to reduce the search space. This step depends on the extraction of stroke features and applying some heuristics to guess the size and location of shapes. The" initial Hypothesis Rejection"process is used when main features of shapes are missed. A stroke consists of a single approximated line can't fire the rectangle hypothesis, and initially this hypothesis is rejected. Fig.5 shows some shape recognition results.

A main problem in shape recognition is the drawing of a single shape using multiple strokes. Many researchers try to simplify the problem by the assumption of single stroke shapes, but practically most of users sketch shapes using many strokes. For complex diagrams, it is required to group stroke into shapes, and the number of combinations is very large. Two types of constraints are used to reduce the number of combinations: 1) the time constraints and 2) the location constraints. The sequence of strokes drawing is available in on-line drawing and usually users finish drawing shapes one by one. Time constraints process utilizes this information, while location constraints process discards the grouping of far strokes.

In fig.6 two multi-strokes shapes are sketched and the sequence of drawing is illustrated. While the search space is complex for only these two shapes, the constraints simplify the search process. Thegroping result is:

Rect (st1,st2,st3) - Triangle (st4,st5).

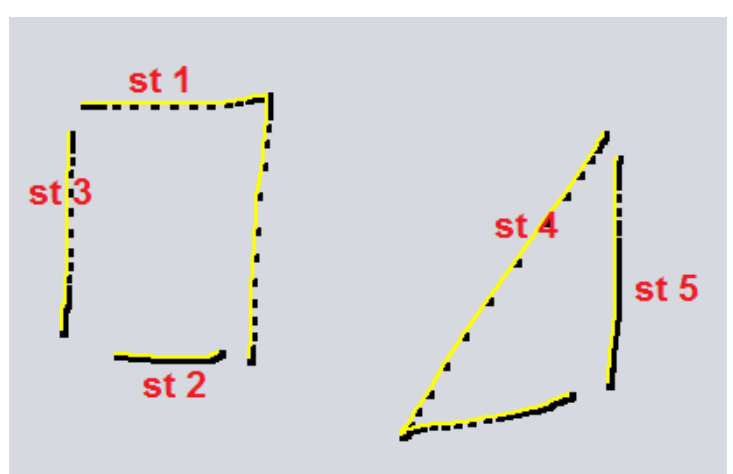

Fig. 6 multi-strokes shapes

\section{IV.DIAGRAM CONSTRUCTION}

The shape recognition phase converts strokes into standard shapes with a limited vision of the relations between different shapes. In diagram layer a wider vision is applied to enhance the arrangement and properties of shapes. In fig.7 (a) hand sketched diagram is recognized perfectly and the result is shown in fig.7 (b). The result diagram has no regularity between different shapes sizes and locations. The user has two choices to adjust this problem, either drawing perfectly, or modify the shapes after recognition. All these solution are tedious and the diagram layer solves these problems and introduces an automated adjustment process.

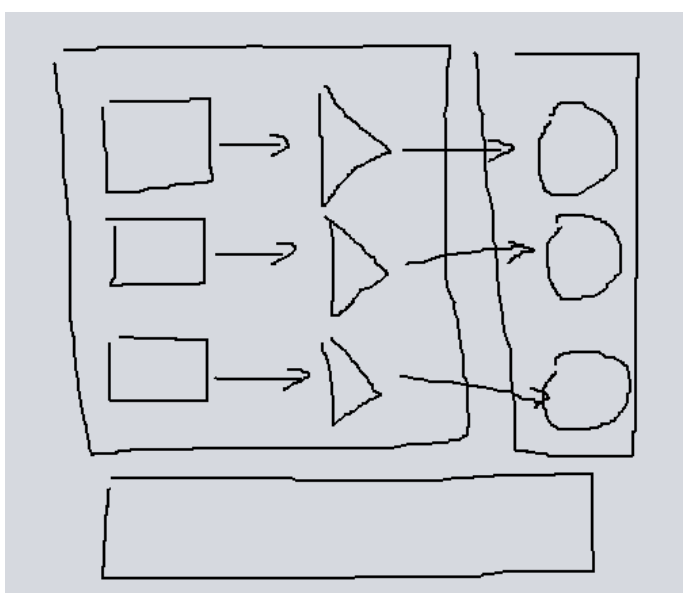

(a) hand sketched diagram

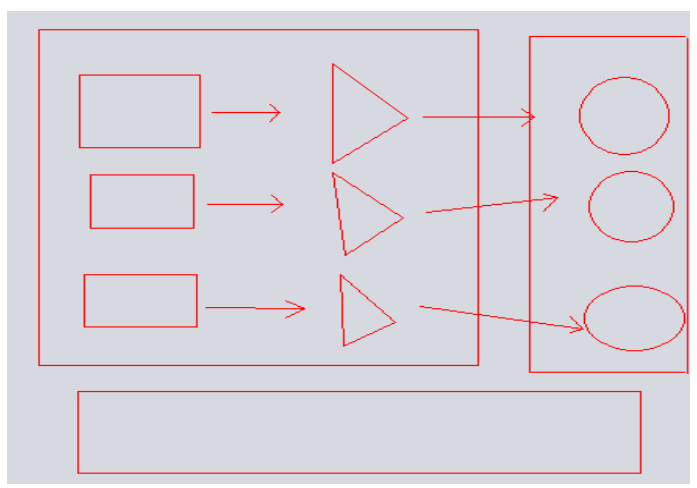

(b) recognition result

Fig. 7 Diagram construction 
Vol. 5, Issue 12, December 2016

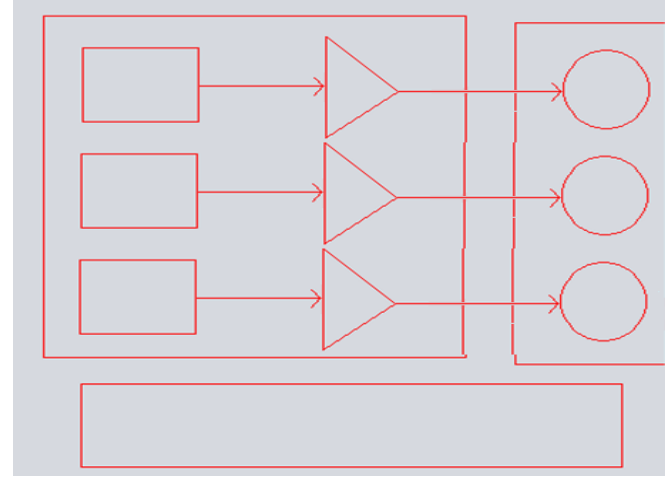

Fig. 8 the rearrangement of shapes

Fig. 8 shows the rearrangement of shapes shown in fig.7 to produce a regular diagram. This process includes many relations between different shapes and requires a set of rules to solve many conflicts and decide which shapes should be modified. The simplest modification is the lines and arrows slopes. When a line is drawn with a slope close to " 0 " or " 90 ",there is a high probability that line is a horizontal or a vertical line. It is required to determine a threshold to which range this correction is valid, to avoid wrong modifications. In addition to that common modification, the repeated shapes with small dimensions deviation has also a probability to be identical shapes. The alignment of shapes is a common correction to arrange shapes in vertical and horizontal rows and columns, and to adjust the starts and ends of lines and arrows.

\section{EXPERIMENTS AND RESULTS}

The developed system is trained to recognize a set of primitive shapes then it is tested using a set of on-line drawn shapes and a set of complex diagrams. Table. 1 lists the primitive shapes and their recognition rate. The performance of shapes rearrangement is not clear in the recognition rates of primitive shapes, but many improvements to the final diagram have been achieved using this system.

\section{VI.CONCLUSION}

Complex diagrams recognition requires not only understanding the primitive shapes but also determining relations between these shapes. Multi-layer framework developed in this system improves the sketch recognition process. The assumption of single stroke shape is usually incorrect and further processing and searching is required to group strokes into shapes. Information available in online drawing helps in reducing the complex search space in multi-stroke matching. The diagram layer has many advantages to improve the final appearance of complex diagram by correcting shapes' sizes and locations.

\begin{tabular}{|c|c|c|}
\hline Shape & Image & Recognition rate \\
\hline Line & & $99 \%$ \\
\hline Arrow & & $96 \%$ \\
\hline
\end{tabular}

\begin{tabular}{|c|c|c|}
\hline Rectangle & & $95 \%$ \\
\hline Square & & \\
\hline Circle & & $97 \%$ \\
\hline Ellipse & & $94 \%$ \\
\hline Triangle & & $93 \%$ \\
\hline Diamond & & $90 \%$ \\
\hline Trapezoid & & \\
\hline
\end{tabular}

\section{REFERENCES}

[1] Andreas Stoffel, Ernesto Tapia, Rául Rojas, Recognition of OnLine Handwritten Commutative Diagrams, 10th International Conference on Document Analysis and Recognition, 2009

[2] Bashir, Rumaan, and Kaiser J. Giri. "Diagram Recognition: Domain Knowledge Based Approach." Machine Intelligence and Research Advancement (ICMIRA), 2013 International Conference on. IEEE, 2013.

[3] E. Lank, J. Thorley, S. Chen, and D. Blostein ,On-line Recognition of UML Diagrams, Proceedings of Sixth International Conference on Document Analysis and Recognition, 2001.

[4] Gennaro Costagliola, Vincenzo Deufemia, Michele Risi ,A Multilayer Parsing Strategy for On-line Recognition of Hand-drawn Diagrams, Visual Languages and Human-Centric Computing ,2006

[5] Lin Wei guo, Jin Xin , A Sketch Recognition Algorithm for Penbased Human-Computer Interaction, 20IO International Conference on Computer Application and System Modeling (ICCASM) 2010

[6] LIU Wei CHA Jian-Zhong, ONLINE HAND-SKET,CHED .e* ENGINEERING DRAWING NEURAL NETWORK RECOGNITION, Proceedings of IEEE TENCON' 2002

[7] Plamondon, R. ; Ecole Polytech., Montreal, Que., Canada ; Srihari, S.N., Online and off-line handwriting recognition: a comprehensive survey, Pattern Analysis and Machine Intelligence, IEEE Transactions on (Volume:22, Issue: 1 ) 2000

[8] Sas, Jerzy, and Urszula Markowska-Kaczmar. "Logical structure recognition of diagram images." Computer Science and Information Systems (FedCSIS), 2015 Federated Conference on. IEEE, 2015

[9] SHI-ZHONG LIAO, WEN-GANG LIU, WEI GUO, COMPOSITE SKETCH SHAPE RECOGNITION BASED ON DAGSVM AND DECISION TREE, Proceedings of the Fifth International Conference on Machine Learning and Cybernetics, Dalian, 13-16 August 2006

[10] T. Hammond, D. Logsdon, B. Paulson, J. Johnston, J. Peschel, A Wolin, and P. Taele, A Sketch Recognition System for Recognizing Free-Hand Course of Action Diagrams, Proceedings of the TwentySecond Innovative Applications of Artificial Intelligence Conference (IAAI-10)

[11] Tracy Hammond, Enabling Instructors to Develop Sketch Recognition Applications for the Classroom, 37th ASEE/IEEE Frontiers in Education Conference ,2007

[12] WEI JIANG1, ZHENG-XING SUN2 HMM-BASED ON-LINE MULTI-STROKE SKETCH RECOGNITION, Proceedings of the Fourth International Conference on Machine Learning and Cybernetics, Guangzhou, 18-21 August 2005

[13] Xinyu Wang, Huosheng $\mathrm{Xu}$, and Heng Wang,On-line Sketch Recognition for Course of Action Diagrams, Proceedings of the International Conference on Mechatronics and Automation IEEE.

[14] Yuan, Zhenming, and Guichao Jin. "Sketch Recognition Based Intelligent Whiteboard Teaching System." Computer Science and Software Engineering, 2008 International Conference on. Vol. 5. IEEE, 2008. 